\section{Avaliação da adequação da assistência pré-natal na rede SUS do Município do Rio de Janeiro, Brasil}

\author{
Adequacy of prenatal care in the National Health \\ System in the city of Rio de Janeiro, Brazil
}

\begin{abstract}
The persistence of negative perinatal outcomes in Rio de Janeiro suggests problems in the quality of prenatal care. The most recent study in the city showed that only $38 \%$ of prenatal care was adequate. This study aimed to evaluate the adequacy of prenatal care under the Brazilian Unified $\mathrm{Na}$ tional Health System in the city of Rio de Janeiro. A cross-sectional study in 2007-2008 interviewed 2,422 women receiving prenatal care for low-risk pregnancy. Evaluation of care used the PHPN index, based on guidelines from the Program for Humanization of Prenatal Care and Childbirth (Brazilian Ministry of Health) and an expanded PHPN index, which included clinical-obstetric procedures, prescription of supplementary ferrous sulfate, and educational activities. According to the PHPN index, 38.5\% of prenatal care was adequate, as compared to $33.3 \%$ based on the expanded PHPN index. Strategies to expand early entry of pregnant women into prenatal care and better use of their contact with the health services in order to promote healthcare measures are essential to correct this situation.
\end{abstract}

Prenatal Care; Program Evaluation; Maternal and Child Health; Unified Health System
Rosa Maria Soares Madeira Domingues 1 Zulmira Maria de Araújo Hartz 2 Marcos Augusto Bastos Dias 3 Maria do Carmo Leal 4

\section{Introdução}

Os desfechos perinatais são resultantes de uma complexa rede de fatores que inclui determinantes biológicos, socioeconômicos e assistenciais 1 . A assistência pré-natal pode contribuir para desfechos mais favoráveis ao permitir a detecção e o tratamento oportuno de afecções, além de controlar fatores de risco que trazem complicações para a saúde da mulher e do bebê.

Revisões sistemáticas demonstram a efetividade de diversas práticas realizadas rotineiramente na assistência pré-natal para prevenção da morbimortalidade materna e perinatal, tais como o diagnóstico e tratamento da hipertensão arterial, anemia, sífilis e infecção urinária; a suplementação de sulfato ferroso; a vacinação antitetânica 2,3,4. Estudos observacionais têm também demonstrado benefícios dessa assistência, geralmente correlacionando maior número de consultas a desfechos mais favoráveis 5,6,7,8,9,10.

No Brasil, observou-se aumento da cobertura da assistência pré-natal e do número de consultas por gestantes nos últimos 15 anos, sendo a proporção de gestantes sem acesso a qualquer consulta de pré-natal inferior a 2\% no ano 2009 (http://www.datasus.gov.br, acessado em 16/ Jun/2011). Entretanto, um estudo que avaliou os óbitos em menores de um ano no Brasil no período 1997-2006 11, utilizando a lista de mortes evitáveis por intervenções do SUS, elaborada por Malta et al. 12, encontrou redução de todas as 
mortes evitáveis, exceto daquelas relacionadas a uma adequada atenção pré-natal, sugerindo baixa qualidade dessa assistência.

No Município do Rio de Janeiro, observa-se situação semelhante, com a persistência de agravos considerados evitáveis, apesar do aumento da cobertura da assistência pré-natal e do número de consultas oferecidas. A mortalidade materna apresenta ainda valores elevados, próximos a 50 por 100 mil nascidos vivos, sendo a hipertensão arterial a principal causa dos óbitos maternos; a mortalidade infantil tem como principal causa as afecções perinatais; observa-se, ainda, incidência elevada de sífilis congênita e de formas graves dessa doença (http://www.saude.rio. rj.gov.br/, acessado em 20/Jun/2010).

O último estudo de avaliação da qualidade da assistência pré-natal representativo para a cidade foi realizado no final da década de 1990. Nessa investigação, em que se utilizou o índice de Kotelchuck modificado, encontrou-se adequação de apenas $38 \%$ dessa assistência 7 .

O índice de Kotelchuck 13, tradicionalmente utilizado para avaliação da assistência pré-natal, mede de forma isolada ou combinada a época de início do acompanhamento e o número de consultas recebidas. Apesar de importante para avaliação da utilização dos serviços de pré-natal, esse índice não permite que se avaliem o conteúdo e a qualidade da assistência. Ademais, utiliza como referência o calendário de consultas preconizado pelo ACOG (American College of Gynecology and Obstetrics) 14, muito diferente do brasileiro, o que tem levado pesquisadores nacionais a adaptá-lo ao calendário de consultas preconizado pelo Ministério da Saúde 7,15.

Nas últimas duas décadas, diversos estudos nacionais têm proposto indicadores de adequação para avaliar a qualidade dos cuidados prénatais, incorporando outros elementos além da época de início do acompanhamento e do número de consultas recebidas 16,17,18. Após a publicação do Programa de Humanização do Pré-Natal e Nascimento (PHPN) pelo Ministério da Saúde 19, que preconiza os parâmetros mínimos para uma adequada assistência pré-natal para gestantes de baixo risco, observa-se a utilização crescente desses parâmetros nos estudos de avaliação 20,21,22,23,24.

Este estudo tem por objetivo avaliar a adequação da assistência pré-natal na rede de serviços do SUS do Município do Rio de Janeiro, segundo práticas consideradas benéficas para os desfechos perinatais contidas nas recomendações do PHPN 19 e nos manuais técnicos do Ministério da Saúde 25.

\section{Material e métodos}

Estudo transversal com gestantes atendidas em unidades de saúde da rede SUS do Município do Rio de Janeiro, realizado no período de novembro de 2007 a julho de 2008. Trata-se de um projeto interinstitucional, coordenado pelo Departamento de Epidemiologia e Métodos Quantitativos em Saúde, Escola Nacional de Saúde Pública Sergio Arouca, Fundação Oswaldo Cruz (ENSP/Fiocruz).

Utilizou-se uma amostragem por conglomerado em dois estágios. No primeiro, foram selecionados os estabelecimentos de saúde com atendimento pré-natal de baixo risco na rede SUS do Município do Rio de Janeiro. Essas unidades primárias de seleção foram estratificadas, segundo tipo de unidade, em: Unidades Básicas de Saúde (UBS), Hospitais/Maternidades, Casa de Parto e Unidades de Saúde da Família (USF).

Dentro dos estratos de UBS e Hospitais, foram consideradas elegíveis as unidades que não estivessem localizadas em área de conflito armado na época da realização do estudo e que apresentassem média mensal de consultas de pré-natal superior a 80 . Foi realizada seleção aleatória simples das unidades de saúde elegíveis nas dez Áreas Programáticas da cidade (AP), divisão territorial e administrativa, conforme critério adotado pela Secretaria Municipal de Saúde do Rio de Janeiro (SMS-RJ), mantendose, na amostra, a mesma distribuição proporcional de unidades por AP existente na época da realização do estudo. No estrato Casa de Parto, a única unidade existente no município foi incluída no estudo.

Considerando a implantação incipiente da Estratégia Saúde da Família na época de realização do estudo, com cobertura populacional de apenas $6 \%$ e ausência de serviços em todas as AP, foi adotado um plano de amostragem distinto para as USF, sendo selecionadas, nas seis áreas da cidade com maior expansão dessa estratégia, as unidades que melhor atendessem aos critérios: (a) não estar localizada em área de conflito armado; (b) dispor do maior número de equipes; (c) ter maior tempo de funcionamento. Considerouse que esses critérios permitiriam a avaliação das unidades que já estivessem com esse modelo assistencial consolidado e viabilizariam a realização do trabalho de campo em menor período de tempo.

No segundo estágio da amostragem, fez-se a seleção das gestantes, sendo consideradas elegíveis todas aquelas em acompanhamento nas unidades incluídas no estudo, independentemente da sua idade, local de residência e época da gestação. As gestantes foram selecionadas 
de forma sistemática, segundo ordem de saída das consultas de pré-natal, em todos os horários de funcionamento dos serviços, até completar a amostra prevista para cada unidade de saúde. Foram realizadas, no máximo, seis entrevistas por turno, com o intuito de garantir a qualidade da entrevista.

O tamanho da amostra foi estabelecido considerando-se o desfecho "adequação do pré-natal”, estimado em $50 \%$, erro alfa de 0,05 , efeito de desenho de 1,5 e correção para população finita, com uma amostra final de 2.417 mulheres. A alocação da amostra foi feita de forma proporcional ao número de consultas pré-natal em cada estrato, observada nos serviços da rede SUS no ano de 2006.

Foram realizadas entrevistas com as gestantes e extraídos dados do cartão de pré-natal. Para a entrevista foi utilizado um questionário padronizado contendo perguntas relativas às condições socioeconômicas, dados demográficos, história reprodutiva materna, dados da gestação atual e informações sobre a assistência recebida. A coleta de dados foi realizada por profissionais e estudantes da área de saúde previamente treinados, sob a supervisão dos pesquisadores, na própria unidade de saúde.

Para obtenção dos dados do cartão de prénatal, foi realizada cópia (manual ou reprográfica) destes. Elaborou-se um instrumento para extração de todas as informações relevantes para avaliação da qualidade da assistência, sendo o seu preenchimento realizado por profissionais de nível superior com experiência na assistência pré-natal. Um instrutivo, padronizando a forma de extração e registro dos dados, foi adotado visando a dar maior homogeneidade na informação obtida e menor viés de aferição. Esses instrumentos foram avaliados e pré-testados no estudo piloto, sendo feitas as modificações necessárias.

Durante o trabalho de campo, todos os questionários foram revisados e codificados por membros da equipe. $\mathrm{O}$ armazenamento dos dados do cartão e da entrevista com a gestante foi realizado por meio do programa Access (Microsoft Corp., Estados Unidos). A digitação foi realizada com dupla entrada em 100\% dos questionários, para avaliação da concordância e verificação de erros. Os problemas identificados foram solucionados com retorno ao questionário ou ao entrevistador, até obter $100 \%$ de concordância.

Para a realização da análise estatística, cada elemento da amostra de gestantes recebeu uma ponderação pelo inverso de sua probabilidade de seleção. Foi realizada análise para amostras complexas, a fim de incorporar o efeito de desenho na análise, uma vez que tem sido demonstrado o impacto na precisão das estimativas quando se utilizam amostras com plano de amostragem por conglomerado 26 .

Para avaliação da adequação da assistência pré-natal, elaborou-se um modelo lógico 27 , apresentado na Figura 1. As áreas sombreadas serão apresentadas e discutidas neste artigo.

A adequação da assistência pré-natal foi verificada utilizando por referência o critério definido pelo PHPN 19, que estabelece um pacote mínimo de procedimentos e exames que devem ser oferecidos a cada gestante: (a) início da assistência pré-natal até o quarto mês de gestação (16 semanas); (b) mínimo de seis consultas, sendo uma no primeiro trimestre gestacional, duas no segundo e três no terceiro; (c) rotina de exames, sendo a inicial solicitada na primeira consulta: tipagem sanguínea, dosagem de hemoglobia (Hg)/hematócrito (Ht), glicemia, VDRL, anti-HIV e exame de urina (EAS), e a seguinte, no início do terceiro trimestre gestacional: VDRL, glicemia e EAS; (d) vacinação antitetânica (VAT); (e) consulta puerperal. Como no estudo não foram incluídas puérperas, o componente consulta puerperal foi excluído.

Foram verificadas também outras práticas contidas no manual Pré-Natal e Puerpério-Atenção Qualificada e Humanizada 25, como (a) procedimentos clínico-obstétricos que devem ser realizados nas consultas de pré-natal: aferição de pressão arterial (PA) e peso, cálculo e registro da idade gestacional (IG), medida da altura de fundo de útero (AFU) e ausculta de batimentos cardiofetais (BCF); (b) prescrição de suplementação de sulfato ferroso; (c) fornecimento de orientações sobre o parto e o aleitamento materno. $\mathrm{O}$ acréscimo desses itens resultou no cálculo do índice PHPN ampliado.

Como no momento da realização do estudo as gestantes estavam em diversas idades gestacionais, foi definido um modelo de avaliação considerando-se o número de consultas e procedimentos que deveriam ter sido realizados até aquele momento da gravidez, segundo as recomendações do Ministério da Saúde, sumarizados na Figura 2.

A definição da IG no início do pré-natal e no momento da entrevista foi realizada de forma padronizada pelos revisores. Para isso, utilizou-se a data da última menstruação (DUM), quando conhecida e confiável, ou a ultrassonografia (USG), realizada preferencialmente até a 14 a semana gestacional.

As informações relativas ao início da assistência pré-natal, número de consultas, resultados dos exames de sangue e urina, procedimentos realizados e vacinação antitetânica foram extraídas do cartão de pré-natal. Por sua vez, as informações sobre solicitação de exames, 
Figura 1

Modelo lógico-operacional para avaliação da implantação da assistência pré-natal.

INSUMOS

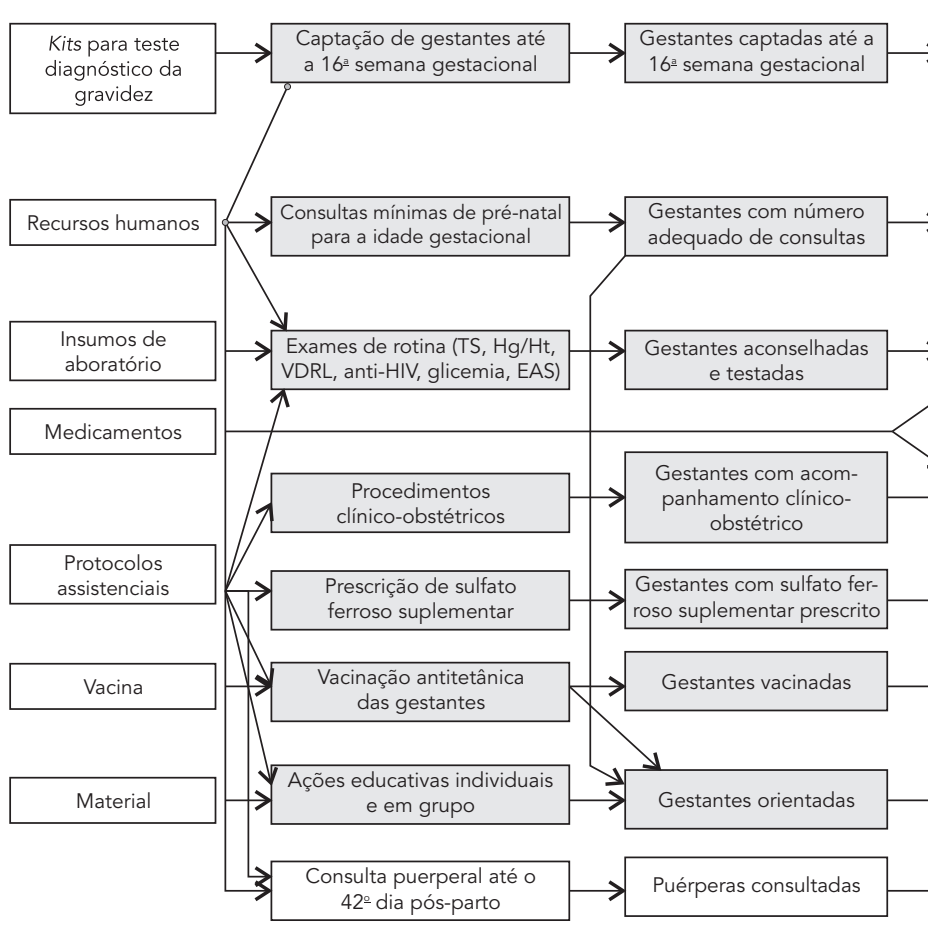

RESULTADOS

IMPACTO

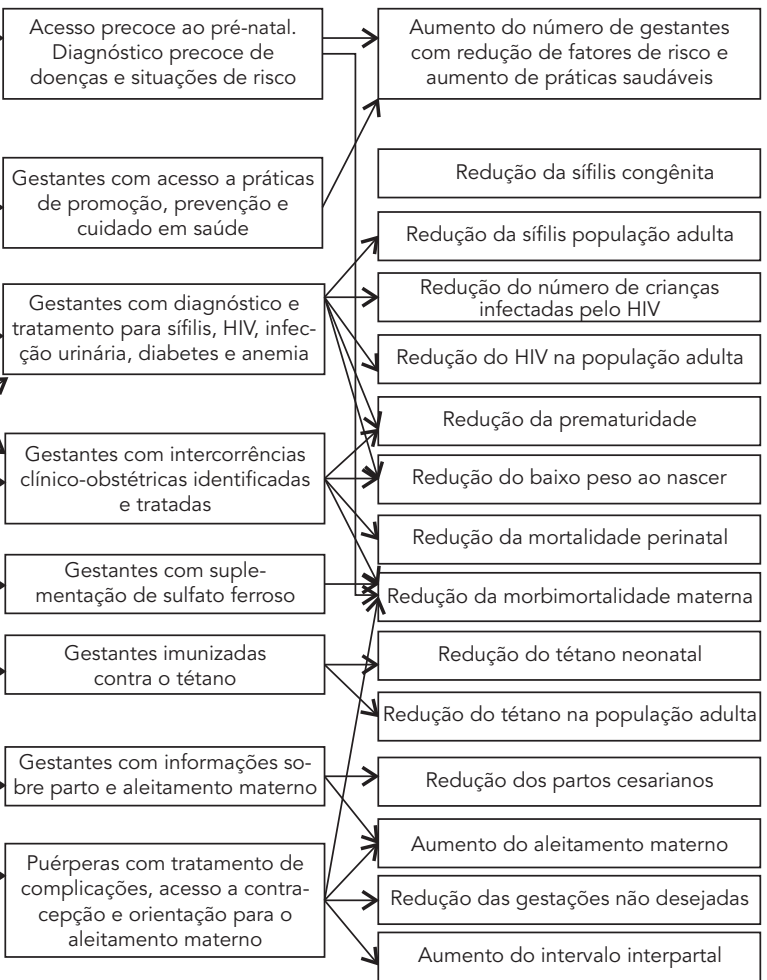

prescrição de sulfato ferroso e orientações recebidas foram obtidas consultando-se a gestante durante a entrevista.

Fez-se a descrição das práticas assistenciais e calculou-se a adequação do PHPN e PHPN ampliado e seus componentes, segundo idade gestacional. Foi realizada a análise bivariada para verificar se a época de início do pré-natal (início até 16 semanas gestacionais) e o número de consultas (adequado ou não para a idade gestacional) estavam associados à adequação dos componentes "exames laboratoriais", "vacinação antitetânica (VAT)", "prescrição de sulfato ferroso suplementar", "orientação sobre o parto” e "orientação sobre o aleitamento", com aplicação do teste qui-quadrado para verificar diferenças entre proporções. A análise dos dados foi realizada por meio do software SPSS 16.0 (SPSS Inc., Chicago, Estados Unidos).

Este projeto foi aprovado pelo Comitê de Ética em Pesquisa da ENSP/Fiocruz, parecer nú- mero 142/06. Os dados foram colhidos mediante assinatura de termo de consentimento livre e esclarecido, apresentado às gestantes após o término de sua consulta de pré-natal, sendo a entrevista realizada logo após a obtenção da anuência. Todos os cuidados foram adotados visando a garantir o sigilo e a confidencialidade das informações obtidas.

\section{Resultados}

Foram entrevistadas 2.422 gestantes, número semelhante ao da amostra calculada, já que as poucas recusas (inferior a 5\%) foram repostas. Das entrevistadas, 69 foram excluídas da análise por não possuírem cartão de pré-natal (25) ou não terem idade gestacional definida no momento da entrevista (44); portanto, foram analisadas 2.353 entrevistas e cartões de pré-natal. As gestantes excluídas da análise não apresentavam diferenças 
Figura 2

Índices PHPN e PHPN ampliado e respectivos componentes, utilizados para avaliação da adequação da assistência pré-natal conforme idade gestacional da gestante no momento da entrevista *.

\begin{tabular}{|c|c|c|c|c|c|c|}
\hline Procedimentos /Idade gestacional & $\begin{array}{l}\text { Até } 16 \\
\text { semanas }\end{array}$ & $\begin{array}{c}17-21 \\
\text { semanas }\end{array}$ & $\begin{array}{c}22-27 \\
\text { semanas }\end{array}$ & $\begin{array}{c}28-33 \\
\text { semanas }\end{array}$ & $\begin{array}{c}34-37 \\
\text { semanas }\end{array}$ & $\begin{array}{c}>37 \\
\text { semanas }\end{array}$ \\
\hline \multicolumn{7}{|l|}{ Parâmetros PHPN ** } \\
\hline \multicolumn{7}{|l|}{$\begin{array}{l}\text { Início pré-natal até a } 16 \text { an semana } \\
\text { gestacional }\end{array}$} \\
\hline Número de consultas & 1 & 2 & 3 & 4 & 5 & 6 \\
\hline \multicolumn{7}{|l|}{ Resultado $1 \underline{\text { a }}$ rotina de exames } \\
\hline \multicolumn{7}{|l|}{ Tipagem sanguínea/Fator Rh } \\
\hline \multicolumn{7}{|l|}{$\mathrm{Hg} / \mathrm{Ht}$} \\
\hline \multicolumn{7}{|l|}{ VDRL } \\
\hline \multicolumn{7}{|l|}{ Anti-HIV } \\
\hline \multicolumn{7}{|l|}{ Glicemia } \\
\hline \multicolumn{7}{|l|}{ EAS } \\
\hline \multicolumn{7}{|l|}{ Resultado 2a rotina de exames } \\
\hline \multicolumn{7}{|l|}{ VDRL } \\
\hline \multicolumn{7}{|l|}{ Glicemia } \\
\hline \multicolumn{7}{|l|}{ EAS } \\
\hline \multicolumn{7}{|l|}{ Vacinação antitetânica } \\
\hline \multicolumn{7}{|l|}{$\begin{array}{l}\text { Vacinação anterior ou } 1 \text { dose na } \\
\text { gestação atual }\end{array}$} \\
\hline \multicolumn{7}{|l|}{$\begin{array}{l}\text { Vacinação anterior ou } 2 \text { doses na } \\
\text { gestação atual }\end{array}$} \\
\hline \multicolumn{7}{|l|}{ 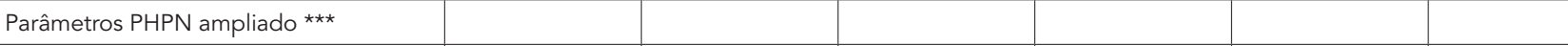 } \\
\hline \multicolumn{7}{|l|}{ Procedimentos nas consultas } \\
\hline Registro da idade gestacional & & $1 \mathrm{ou}+$ & $2 \mathrm{ou}+$ & 3 ou + & $4 \mathrm{ou}+$ & $5 \mathrm{ou}+$ \\
\hline Registro da pressão arterial & $1 \mathrm{ou}+$ & $2 \mathrm{ou}+$ & 3 ou + & 4 ou + & 5 ou + & 6 ou + \\
\hline Registro do peso da gestante & $1 \mathrm{ou}+$ & 2 ou + & 3 ou + & 4 ou + & 5 ou + & 6 ou + \\
\hline Registro da altura uterina & & & $1 \mathrm{ou}+$ & 2 ou + & 3 ou + & 4 ou + \\
\hline Registro dos batimentos cardiofetais & & & $1 \mathrm{ou}+$ & $2 \mathrm{ou}+$ & 3 ou + & 4 ou + \\
\hline \multicolumn{7}{|l|}{$\begin{array}{l}\text { Prescrição de sulfato ferroso } \\
\text { suplementar }\end{array}$} \\
\hline \multicolumn{7}{|l|}{ Orientações sobre o parto\# } \\
\hline $\begin{array}{l}\text { Orientações sobre aleitamento } \\
\text { materno \#\# }\end{array}$ & & & & & & \\
\hline
\end{tabular}

EAS: elementos anormais e sedimentos na urina; Hg: hemoglobina; Ht: hematócrito; PHPN: Programa de Humanização do Pré-Natal e Nascimento.

* Considerada assistência adequada se gestante apresentava os procedimentos previstos para a idade gestacional, conforme assinalado no quadro acima;

** Critério PHPN: início do pré-natal até a 16ạ semana gestacional, número mínimo de consultas para a idade gestacional, resultado da 1ạ rotina de exames a partir da 22a semana gestacional, vacinação antitetânica a partir da 28a semana gestacional, resultado da segunda rotina de exames após a 34a semana gestacional:

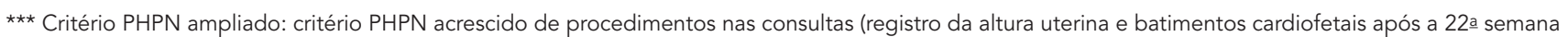
gestacional), prescrição de sulfato ferroso suplementar após a 22a semana gestacional e orientações sobre parto e amamentação após a 34a semana gestacional;

\# Consideradas orientações sobre o parto: orientações sobre sinais do trabalho de parto + direito ao acompanhante + importância de levar cartão de pré-natal para a maternidade;

\#\# Considerada orientação sobre amamentação se gestante referia que algum profissional conversou com ela sobre o tema. 
significativas em relação às características socioeconômicas e demográficas das demais, observando-se apenas proporção um pouco menor de primigestas (Tabela 1).

Na Tabela 2, são apresentadas as práticas assistenciais encontradas segundo faixa gestacional no momento da entrevista. É mostrada também a adequação global e por componente do PHPN e do PHPN ampliado, em cada faixa gestacional e para o conjunto das gestantes entrevistadas.

A mediana da idade gestacional no início do pré-natal foi de 13 semanas, com variação de 4 a 39. Do total de entrevistadas, $74,4 \%$ apresentaram início precoce da assistência pré-natal, realizada até a 16a semana gestacional, e apenas $2 \%$ iniciaram o pré-natal no terceiro trimestre gestacional.

De acordo com o calendário preconizado pelo Ministério da Saúde, o número de consultas só foi adequado para as gestantes que estavam no início da gestação. Observou-se um pequeno aumento da adequação do número de consultas no final da gravidez, alcançando valor próximo a 80\% (Tabela 2).

O registro, no cartão de pré-natal, dos procedimentos realizados em cada consulta foi elevado, principalmente no que se refere à aferição do peso e da pressão arterial, informação confirmada por $96,3 \%$ das gestantes, as quais relataram que esses procedimentos foram realizados em todas as consultas (dado não apresentado em Tabela). O registro da IG, da AFU e do BCF apresentou valores menores, próximos a $80 \%$ (Tabela 2).

Apesar do aumento do registro dos resultados de exames com o avançar da gravidez, a adequação atingiu um valor máximo de apenas $41 \%$ em gestantes com $28-33$ semanas. Nas gestantes com 34-37 semanas, quando já deveriam estar disponíveis os resultados dos exames da segunda rotina, observou-se uma queda da adequação, que não alcançou $20 \%$ nas gestantes a termo. Entre aquelas com mais de 37 semanas,

Tabela 1

Características socioeconômicas, demográficas e reprodutivas das gestantes incluídas e excluídas da análise. Município do Rio de Janeiro, Brasil, 2007-2008.

\begin{tabular}{|c|c|c|c|c|c|}
\hline Características das gestantes & $\begin{array}{l}\text { Incluídas na análise } \\
\qquad(n=2.353)\end{array}$ & $\%$ & $\begin{array}{l}\text { Excluídas da análise } \\
\qquad(\mathrm{n}=69)\end{array}$ & $\%$ & Valor de $p$ * \\
\hline \multicolumn{6}{|l|}{ Idade (anos) } \\
\hline Até 19 & 554 & 23,6 & 14 & 20,1 & \\
\hline $20-34$ & 1.597 & 67,9 & 45 & 65,4 & \\
\hline 35 e + & 201 & 8,5 & 10 & 14,5 & 0,069 \\
\hline \multicolumn{6}{|l|}{ Cor da pele } \\
\hline Branca & 582 & 25,4 & 22 & 31,8 & \\
\hline Parda & 1.171 & 51 & 32 & 45,9 & \\
\hline Preta & 542 & 23,6 & 15 & 22,3 & 0,389 \\
\hline \multicolumn{6}{|l|}{ Escolaridade } \\
\hline Fundamental incompleto & 798 & 33,9 & 27 & 38,6 & \\
\hline Fundamental completo & 754 & 32 & 21 & 30,1 & \\
\hline Ensino Médio & 801 & 34 & 22 & 31,3 & 0,668 \\
\hline Trabalho remunerado & 818 & 34,8 & 23 & 34,1 & 0,907 \\
\hline Vive com companheiro & 1.829 & 77,7 & 49 & 71,8 & 0,285 \\
\hline \multicolumn{6}{|l|}{ Local de atendimento } \\
\hline Hospital & 841 & 35,8 & 26 & 37,1 & \\
\hline UBS & 1.327 & 56,4 & 36 & 52,1 & \\
\hline USF/Casa de Parto & 185 & 7,8 & 7 & 10,8 & 0,839 \\
\hline Primigesta & 880 & 37,4 & 15 & 21,3 & 0,009 \\
\hline Antecedente de doença crônica ** & 190 & 8,1 & 9 & 12,7 & 0,091 \\
\hline 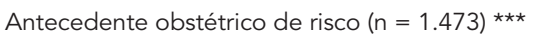 & 544 & 36,9 & 18 & 33,4 & 0,522 \\
\hline
\end{tabular}

UBS: unidade básica de saúde; USF: unidade de saúde da família.

* Teste qui-quadrado;

** Antecedente de hipertensão arterial crônica ou diabetes não gestacional;

*** Apenas em mulheres com gestação anterior. 
Distribuição proporcional de procedimentos preconizados na assistência pré-natal e sua adequação, considerando-se idade gestacional no momento da entrevista, conforme índices PHPN e PHPN ampliado. Município do Rio de Janeiro, Brasil, 2007-2008.

\begin{tabular}{|c|c|c|c|c|c|c|c|}
\hline Procedimentos/idade gestacional & $\begin{array}{l}\text { Até } 16 \\
\text { semanas } \\
(n=355)\end{array}$ & $\begin{array}{c}17-21 \\
\text { semanas } \\
(n=342)\end{array}$ & $\begin{array}{l}22-27 \\
\text { semanas } \\
(n=448)\end{array}$ & $\begin{array}{c}28-33 \\
\text { semanas } \\
(n=531)\end{array}$ & $\begin{array}{c}34-37 \\
\text { semanas } \\
(n=459)\end{array}$ & $\begin{array}{c}>37 \\
\text { semanas } \\
(n=218)\end{array}$ & $\begin{array}{c}\text { Total * } \\
(\mathrm{n}=2.353)\end{array}$ \\
\hline \multicolumn{8}{|l|}{ Parâmetros PHPN } \\
\hline Adequação início pré-natal & 100,0 & 73,2 & 70,2 & 70,2 & 66,7 & 69,5 & 74,4 \\
\hline Adequação número de consultas & 100,0 & 77,3 & 69,9 & 74,3 & 77,8 & 82,0 & 79,2 \\
\hline \multicolumn{8}{|l|}{ Resultado da 1a rotina de exames } \\
\hline Tipagem sanguínea & 27,8 & 54,0 & 65,1 & 76,6 & 83,9 & 89,4 & \\
\hline $\mathrm{Hg} / \mathrm{Ht}$ & 21,9 & 39,6 & 54,8 & 65,5 & 72,4 & 77,1 & \\
\hline VDRL & 23,2 & 47,3 & 62,8 & 75,9 & 80,9 & 89,8 & \\
\hline Anti-HIV & 9,5 & 26,6 & 43,4 & 59,2 & 67,7 & 74,0 & \\
\hline Glicemia & 21,3 & 37,1 & 53,3 & 62,2 & 68,9 & 73,5 & \\
\hline EAS & 17,9 & 31,7 & 46,9 & 57,0 & 62,3 & 65,0 & \\
\hline \multicolumn{8}{|l|}{ Resultado da 2a rotina de exames } \\
\hline VDRL & & & & & 31,3 & 35,3 & \\
\hline Glicemia & & & & & 33,2 & 36,6 & \\
\hline EAS & & & & & 25,0 & 25,5 & \\
\hline Adequação exames (1ạ e 2ạ rotina) & & & 30,5 & 41,1 & 17,0 & 19,3 & \\
\hline Adequação vacinação antitetânica & & & & 54,8 & 55,1 & 54,2 & \\
\hline \multicolumn{8}{|l|}{ Parâmetros PHPN ampliado } \\
\hline \multicolumn{8}{|l|}{ Registro procedimentos ** } \\
\hline Idade gestacional & 86,8 & 82,9 & 78,8 & 77,1 & 78,8 & 73,1 & 79,7 \\
\hline Pressão arterial & 95,8 & 95,7 & 94,4 & 94,4 & 92,1 & 92,8 & 94,2 \\
\hline Peso da gestante & 95,7 & 95,9 & 95,4 & 94,9 & 90,9 & 89,1 & 94,0 \\
\hline Altura uterina & & & 86,0 & 86,8 & 81,5 & 80,2 & \\
\hline Batimentos cardiofetais & & & 80,5 & 79,0 & 76,2 & 76,8 & \\
\hline Adequação procedimentos & 97,6 & 70,1 & 56,1 & 61,5 & 63,8 & 69,4 & \\
\hline Adequação sulfato ferroso *** & & & 84,5 & 89,0 & 88,5 & 90,8 & \\
\hline \multicolumn{8}{|l|}{ Orientações sobre o parto } \\
\hline Sinais de trabalho de parto & & & & & 40,3 & 51,0 & \\
\hline Direito ao acompanhante & & & & & 33,2 & 36,4 & \\
\hline $\begin{array}{l}\text { Importância de levar cartão para } \\
\text { maternidade }\end{array}$ & & & & & 72,8 & 75,5 & \\
\hline Referência para maternidade & & & & & 43,6 & 59,5 & \\
\hline $\begin{array}{l}\text { Adequação orientações sobre o } \\
\text { parto }\end{array}$ & & & & & 23,1 & 23,3 & \\
\hline $\begin{array}{l}\text { Adequação orientações } \\
\text { aleitamento materno }\end{array}$ & & & & & 55,1 & 50,9 & \\
\hline Adequação PHPN & 100,0 & 73,2 & 27,8 & 21,8 & 9,0 & 8,6 & 38,5 \\
\hline Adequação PHPN ampliado & 97,6 & 66,4 & 20,8 & 18,4 & 2,8 & 2,2 & 33,3 \\
\hline
\end{tabular}

EAS: elementos anormais e sedimentos na urina; Hg: hemoglobina; Ht: hematócrito; PHPN: Programa de Humanização do Pré-Natal e Nascimento.

* Total informado apenas para procedimentos ou exames exigidos em todas as idades gestacionais;

** Proporção de gestantes com registro do procedimento em todas as consultas de pré-natal;

*** Prescrição de sulfato ferroso suplementar em mulheres sem diagnóstico de anemia. 
a maior proporção de registro foi observada para a tipagem sanguínea eVDRL (próximo a 90\%), na primeira rotina, e VDRL e glicemia, na segunda (36\%). A menor proporção de registros, tanto na primeira quanto na segunda rotina de exames, foi observada para os resultados do exame de urina (EAS) (Tabela 2).

Ressalta-se que a solicitação de exames de sangue e urina foi elevada, sendo referida por mais de 98\% das gestantes (dado não apresentado em tabela). Excluindo-se aquelas que haviam recebido o pedido do exame no dia da entrevista, menos de $5 \%$ das grávidas referiram não ter feito o exame, sendo os valores semelhantes para os exames de sangue e urina.

Quanto à vacinação antitetânica, também se observou um aumento do registro de doses à medida que aumentou a idade gestacional das mulheres entrevistadas, porém sem atingir a cobertura desejada. Em mulheres com mais de 37 semanas, apenas $54 \%$ poderiam ser consideradas imunizadas para a prevenção do tétano neonatal (Tabela 2).

A prescrição de suplementação de sulfato ferroso, feita para mulheres sem diagnóstico de anemia, foi elevada, próxima a $90 \%$ em todas as faixas gestacionais (Tabela 2).

As orientações para o parto apresentaram valores crescentes com o avançar da gestação, sendo ainda bastante restritas. A orientação mais recebida foi sobre a importância de levar o cartão de pré-natal para a maternidade no momento do parto, seguida pelas informações sobre sinais de início do trabalho de parto e direito a um acompanhante durante a internação. As orientações foram fragmentadas e menos de $25 \%$ das entrevistadas referiam ter recebido as três orientações. Das gestantes que já se encontravam no último mês de gestação, $40 \%$ ainda não haviam recebido informação sobre qual maternidade procurar no momento do parto. As orientações sobre amamentação foram recebidas por aproximadamente $50 \%$ das gestantes (Tabela 2).

Considerando apenas os parâmetros do PHPN, 38,5\% das gestantes apresentavam assistência adequada, observando-se uma redução da adequação de $100 \%$ naquelas com até 16 semanas gestacionais a apenas $8,6 \%$ para as com mais de 37 semanas. Para o critério PHPN ampliado, a adequação se reduziu para $33,3 \%$, sendo de apenas $2,2 \%$ para as gestantes a termo (Tabela 2).

$\mathrm{Na}$ análise bivariada, o início precoce da assistência pré-natal esteve associado à maior adequação de consultas, sendo a proporção de gestantes com número adequado de consultas três vezes maior em quem iniciou acompanhamento pré-natal antes da 16a semana. Tanto o início precoce quanto o número adequado de consultas estiveram associados à maior adequação de realização de exames e de vacinação antitetânica, estando o número de consultas associado também à maior orientação em relação ao parto e à amamentação (Tabela 3).

\section{Discussão}

Este estudo teve por objetivo avaliar a adequação da assistência pré-natal na rede SUS do Município do Rio de Janeiro, tendo sido encontrados inúmeros problemas no processo de assistência pré-natal, considerando-se os parâmetros preconizados pelo PHPN.

O primeiro foi o início tardio da assistência pré-natal, após o quarto mês de gestação, observado em aproximadamente $25 \%$ das gestantes. O início precoce da assistência pré-natal permite o acesso a métodos diagnósticos e terapêuticos para diversas patologias com repercussões graves para a saúde da mulher e do bebê, tais como hipertensão arterial crônica, diabetes não gestacional, anemia, infecção pela sífilis e pelo HIV. Além disso, propicia uma estimativa da idade gestacional mais precisa, com melhor monitoramento do crescimento fetal e melhor embasamento para decisões relacionadas a uma possível interrupção da gravidez. Vale lembrar que o protocolo assistencial de outros países, como Estados Unidos 14 e Inglaterra 28, recomendam o início do pré-natal até a 12a semana gestacional, o que elevaria a inadequação observada para aproximadamente $50 \%$.

O resultado encontrado foi pior do que o relatado por Leal et al. 7 em estudo realizado no Município do Rio de Janeiro em 1999, provavelmente em decorrência da inclusão de gestantes de melhor nível socioeconômico atendidas no setor privado não conveniado ao SUS no primeiro estudo. Outros estudos nacionais também encontraram problemas na captação precoce de gestantes, com valores que variaram de $14 \%$ a $82 \% 15,16,17,20,21,23,24,29$. Entretanto, é possível que esses resultados tenham determinantes diferentes, relacionadas às características das gestantes e da organização da rede de serviços de cada localidade.

Além de ser um importante componente da adequação do pré-natal, verificou-se que o início tardio afetou a adequação do número de consultas, sendo a proporção de gestantes com número adequado de consultas muito menor entre aquelas com início do pré-natal após a 16a semana gestacional. Esse resultado sugere a ausência de mecanismos compensatórios que poderiam ser utilizados pelos serviços a fim de garantir o acesso ao número mínimo de consultas preconizado, 
Análise bivariada dos componentes do critério PHPN e PHPN ampliado *. Município do Rio de Janeiro, Brasil, $2007-2008$.

\begin{tabular}{|c|c|c|c|c|c|c|c|c|c|c|c|c|}
\hline $\begin{array}{l}\text { Exposição/ } \\
\text { Desfecho }\end{array}$ & $\begin{array}{l}\text { Adequa- } \\
\text { ção número } \\
\text { consultas } \\
\text { (n = 2.353) }\end{array}$ & $\begin{array}{l}\text { Valor } \\
\text { de } p\end{array}$ & $\begin{array}{c}\text { Adequação } \\
\text { de exames } \\
\star \star\end{array}$ & $\begin{array}{l}\text { Valor } \\
\text { de } p\end{array}$ & $\begin{array}{l}\text { Adequação } \\
\text { vacinação } \\
(n=1.208)\end{array}$ & $\begin{array}{l}\text { Valor } \\
\text { de p }\end{array}$ & $\begin{array}{c}\text { Adequação } \\
\text { sulfato } \\
\text { ferroso } * * * \\
(n=1.072)\end{array}$ & $\begin{array}{l}\text { Valor } \\
\text { de } p\end{array}$ & $\begin{array}{l}\text { Orientações } \\
\text { sobre o } \\
\text { parto } \\
(n=677)\end{array}$ & $\begin{array}{l}\text { Valor } \\
\text { de } p\end{array}$ & $\begin{array}{l}\text { Orientações } \\
\text { sobre } \\
\text { aleitamento } \\
\text { materno } \\
\text { (n=677) }\end{array}$ & $\begin{array}{l}\text { Valor } \\
\text { de } p\end{array}$ \\
\hline
\end{tabular}

\begin{tabular}{|c|c|c|c|c|c|c|c|c|c|c|c|c|}
\hline \multicolumn{13}{|c|}{ Início até } \\
\hline \multicolumn{13}{|c|}{ gestacionais } \\
\hline Sim & 95,6 & & 34,3 & & 57,6 & & 88,6 & & 25,2 & & 53,6 & \\
\hline Não & 31,7 & $<0,001$ & 16,2 & $<0,001$ & 49,1 & 0,006 & 86,6 & 0,589 & 19,3 & 0,061 & 54,0 & 0,914 \\
\hline \multicolumn{13}{|c|}{ Adequação } \\
\hline \multicolumn{13}{|c|}{ número de } \\
\hline \multicolumn{13}{|c|}{ consultas } \\
\hline Sim & & & 35,1 & & 59,5 & & 89 & & 26 & & 56,2 & \\
\hline Não & & & 9,4 & $<0,001$ & 39,1 & $<0,001$ & 84,5 & 0,232 & 12,4 & 0,03 & 44,5 & 0,029 \\
\hline
\end{tabular}

PHPN: Programa de Hmanização do Pré-Natal e Nascimento.

* Teste estatístico qui-quadrado;

** Considerada apenas a adequação da primeira rotina de exames em gestantes com 22 ou mais semanas gestacionais;

*** Gestantes com 22 ou mais semanas gestacionais sem diagnóstico de anemia.

como, por exemplo, adotar intervalos mais curtos entre as consultas.

Destaca-se que a adequação do número de consultas foi superior à adequação do início precoce, sendo observado um aumento da adequação de consultas no final da gestação. Esse resultado é provavelmente decorrente de uma maior realização de consultas no último trimestre gestacional, quando o intervalo entre as consultas é menor, não tendo sido possível verificar se as consultas foram realizadas conforme distribuição recomendada pelo Ministério da Saúde.

A baixa cobertura de consultas também foi identificada em outros estudos nacionais $21,22,24,29$, assim como problemas na distribuição destas conforme o calendário preconizado pelo Ministério da Saúde 17.

Existem evidências de que a redução do número de consultas de pré-natal não acarreta piores resultados maternos e perinatais, embora as mulheres, principalmente em países desenvolvidos, refiram menor satisfação com o modelo de atenção com número reduzido de consultas 3 . Neste estudo, gestantes com menor número de consultas apresentaram menor adequação de exames, vacinação e orientação sobre o parto e a amamentação. Associação negativa entre número de consultas e realização de exames e procedimentos também foi verificada em outros estudos 15,18,30,31.
A definição do número ideal de consultas é importante para a elaboração de protocolos assistenciais e planejamento dos recursos em saúde. Teoricamente, um número maior de consultas pode significar mais oportunidades de receber cuidados preventivos e de promoção de saúde, especialmente em gestações de maior risco, com maior probabilidade de afetar os desfechos perinatais 9 . No entanto, a discussão mais relevante é sobre a qualidade dessas consultas e a utilização desses contatos com os serviços para a realização de cuidados efetivos em saúde.

Neste trabalho, a realização dos procedimentos de aferição da pressão arterial e peso foi elevada, próxima a $100 \%$, semelhante à observada em outros locais 16,17,18,21,23. Já o registro do cálculo da idade gestacional, medição da AFU e do BCF, importantes para o monitoramento do crescimento e bem-estar fetal, apresentou proporções mais baixas, próximas a $80 \%$, sugerindo maior incorporação dos primeiros à rotina de pré-natal. Em adição, além de realizá-los, é importante verificar se os procedimentos têm sido utilizados para o diagnóstico e tratamento adequado de intercorrências gestacionais. Uma avaliação do manejo de gestantes com hipertensão arterial em unidades de saúde da rede SUS do Rio de Janeiro revelou diversos problemas no processo de cuidado, relacionados principalmente à conduta dos profissionais de saúde ${ }^{32}$. 
A realização dos exames é outro componente do pré-natal que aponta perdas de oportunidades de intervenção. A solicitação e coleta de exames foram elevadas e, ainda que informações específicas de cada exame não estivessem disponíveis, sugerem adesão dos profissionais e das mulheres aos exames de rotina. Contudo, a proporção de gestantes com registro de resultados de exames foi baixa, inferior a $100 \%$, mesmo em gestantes a termo. Embora o problema possa ser apenas de registro dos resultados, há também a possibilidade de falhas na execução dos exames, por falta de insumos específicos, equipamentos danificados, demora no retorno do resultado, extravio das amostras, entre outros. Por essa razão, a falta do registro no cartão foi considerada como não realização do procedimento e consequente inadequação do cuidado pré-natal, critério já adotado em outros estudos de avaliação 16,17,30.

Os valores encontrados para exames de sangue, tomando-se por referência o registro do resultado da primeira rotina em gestantes com mais de 37 semanas, foram semelhantes aos encontrados em outros estudos realizados com puérperas que verificaram a realização do exame em cartões ou prontuários de pré-natal 16,17,18,21. $\mathrm{O}$ registro de exame de urina foi o que alcançou menor valor, sendo muito inferior ao observado em estudo realizado no Sul do país, onde a cobertura foi próxima a $100 \% 31$. Ressalta-se a baixa proporção de resultados dos exames da segunda rotina, disponível para apenas um terço das gestantes a termo. Serruya et al. 22, em avaliação dos primeiros anos de implantação do PHPN no Brasil, verificaram que ao adicionar toda a rotina de exames, incluindo a realização do anti-HIV, a adequação obtida foi inferior a 5\%.

A ausência do resultado de exames básicos de rotina para $100 \%$ das gestantes representa a perda de oportunidade de diagnóstico e tratamento de agravos passíveis de controle, como a infecção pela sífilis e pelo HIV, anemia, infecção urinária e bacteriúria assintomática, causas de vários desfechos perinatais negativos e para as quais existem intervenções efetivas 2. Acrescente-se, ainda, o desperdício de recursos, uma vez que os exames foram realizados, sem que o resultado retornasse em tempo hábil.

Quanto à vacinação, os valores encontrados foram bem menores que os preconizados, sendo inferiores ao relatado por outros autores 17,20,30. Considerando a elevada cobertura vacinal no Município do Rio de Janeiro, é pouco provável que esse resultado seja decorrente da falta do imunobiológico específico nas unidades de saúde. Baixa adesão dos profissionais e/ou das gestantes ao protocolo de vacinação e problemas no registro da vacinação no cartão da gestante são fatores que deveriam ser mais bem investigados para orientar estratégias de ação.

A prescrição de sulfato ferroso suplementar foi elevada e superior à encontrada em outros estudos nacionais 16,21,23,29, evidenciando a boa adesão dos profissionais a essa prática.

Por sua vez, as orientações sobre o parto e a amamentação foram referidas por uma proporção pequena de mulheres, mesmo entre aquelas com mais de 37 semanas e já próximas à data do parto. Esse resultado assemelha-se ao encontrado na avaliação de outros serviços 15,17,29,33,34.

A orientação sobre o direito ao acompanhante foi a menos frequente. A presença do acompanhante é uma prática reconhecidamente benéfica para assistência ao parto, estando associada a diversos desfechos favoráveis 35. É um direito das gestantes garantido por lei e regulamentado pelo SUS (Lei Federal $n^{\circ}$. 11.108 de 7 de abril de 2005). Em estudo anterior, realizado em maternidade pública localizada no Município do Rio de Janeiro, uma das razões encontradas para a ausência do acompanhante no parto foi o desconhecimento das gestantes sobre esse direito, sendo fundamental o papel do serviço de prénatal nesse esclarecimento 33 .

A baixa proporção de mulheres que receberam informação sobre qual maternidade procurar no momento do parto é provavelmente decorrente da inexistência de um sistema de referência formal para o parto na cidade do Rio de Janeiro, na ocasião em que o estudo foi realizado. Em pesquisa realizada em Curitiba, onde a vinculação para uma maternidade de referência é um dos componentes do Programa Mãe Curitibana, a orientação foi relatada por $98,3 \%$ das gestantes 17 . Deve-se ressaltar que a vinculação da gestante para a maternidade na qual será realizado o seu parto é um direito das mulheres assistidas pelo SUS, garantido pela Lei $n^{o}$. 11.634 de 27 de dezembro de 2007, e tem como um de seus objetivos evitar a peregrinação das gestantes no momento da internação para assistência ao parto.

As orientações sobre aleitamento, embora um pouco mais frequentes do que as relacionadas ao parto, foram também baixas, sendo semelhantes 15,29 ou inferiores 17,20 ao que se encontrou em estudos realizados em outros municípios brasileiros. A orientação e preparação para o aleitamento, realizada durante a assistência pré-natal, é considerada fundamental para o sucesso da prática da amamentação, sendo um dos dez passos preconizados pelas Iniciativas Hospital Amigo da Criança e Unidade Básica Amiga da Amamentação 36 .

A adequação global da assistência pré-natal foi baixa, menor que $10 \%$ em gestantes com mais de 37 semanas, resultado semelhante ou inferior 
aos dos estudos que utilizaram o PHPN, ou índices similares, em suas avaliações com puérperas 16,17,18,20,21,23,24. Em todas essas investigações, a proporção de adequação se reduziu quando foram acrescidos outros itens ao início precoce e número de consultas, principalmente a realização completa dos exames de rotina.

Análises com base no número de consultas de pré-natal podem esconder graves problemas na qualidade do cuidado ofertado, sendo uma explicação possível para as inconsistências nos resultados de avaliações da efetividade do cuidado pré-natal que utilizam apenas esse critério 9,37,38. A inadequação da assistência pré-natal pode estar associada tanto à não realização de procedimentos, quanto à utilização excessiva destes. Barros et al. 38, comparando os dados das três coortes realizadas na cidade de Pelotas, Rio Grande do Sul, identificaram um aumento da proporção de partos prematuros, os quais poderiam estar sendo determinados pelo uso excessivo de intervenções desnecessárias, dentre eles os exames de ultrassonografia durante a assistência pré-natal.

A utilização do critério PHPN, ao incorporar outros componentes além do número de consultas, representa um avanço na avaliação do conteúdo da assistência pré-natal. Todavia, o indicador não contempla todos os procedimentos efetivos que poderiam ser realizados na assistência pré-natal, ainda que alguns estejam recomendados nos manuais técnicos do Ministério da Saúde. Dessa forma, uma avaliação que indique adequação da assistência segundo o PHPN pode encobrir falhas na assistência que acarretem desfechos perinatais negativos, subestimando a efetividade do pré-natal.

No presente estudo, foi necessário adaptar o critério PHPN ao momento gestacional de cada entrevistada, sendo possível que essa forma de aferição da adequação tenha resultado em valores diferentes do que seria obtido ao término da gestação, quando, de fato, a assistência já estaria finalizada. Entretanto, a inclusão de gestantes de várias idades gestacionais pode ter reduzido o viés de memória para fatos ocorridos no início da gestação, passíveis de ocorrer em pesquisas realizadas com puérperas. Ademais, a verificação dos procedimentos nas diversas faixas gestacionais permitiu visualizar o cumprimento do protocolo recomendado ao logo da gestação, e não apenas seu resultado final, simulando um estudo longitudinal, apesar do seu desenho transversal.

Para concluir, podemos afirmar que a assistência pré-natal na rede SUS da cidade do Rio de Janeiro, em seus componentes mais básicos preconizados pelo PHPN, apresentou inúmeras falhas, resultando numa adequação muita baixa da assistência, principalmente das gestantes a termo. Esse achado pode explicar a persistência de resultados perinatais desfavoráveis, apesar do aumento da cobertura pré-natal na cidade. Destaca-se que esses resultados não podem ser aplicados a unidades com número pequeno de atendimentos, localizadas em áreas de conflito e com menor tempo de funcionamento, em decorrência da exclusão de serviços com essas características na seleção da amostra. Nesse caso, é provável que essas unidades apresentem adequação ainda menor do pré-natal.

A ampliação do acesso ao início precoce da assistência pré-natal e a melhor utilização dos contatos com os serviços de saúde para a realização de cuidados efetivos em saúde, tanto em atendimentos individuais, quanto coletivos, revelam-se prioritários na reversão desse quadro. 


\section{Resumo}

A persistência de desfechos perinatais negativos no Município do Rio de Janeiro, Brasil, sugere problemas na qualidade da assistência pré-natal. A última investigação realizada nessa cidade mostrou adequação de apenas 38\% dessa assistência. O objetivo deste estudo é avaliar a adequação da assistência pré-natal na rede do SUS do Município do Rio de Janeiro. Foi realizado um estudo transversal, em 2007-2008, por meio de entrevistas com 2.422 gestantes em atendimento nos serviços de pré-natal de baixo risco. Para avaliação da adequação da assistência, foi utilizado o índice PHPN, com as recomendações do Programa de Humanização do Pré-natal e Nascimento, do Ministério da Saúde, e um índice PHPN ampliado, em que foram acrescentados procedimentos clinico-obstétricos, prescrição de sulfato ferroso suplementar e ações educativas. Fo $i$ encontrada adequação de 38,5\% para o PHPN e 33,3\% para o PHPN ampliado. Estratégias de ampliação da captação precoce das gestantes e melhor utilização dos contatos com os serviços para a realização de ações de atenção à saúde são prioritárias para a reversão desse quadro.

Cuidado Pré-Natal; Avaliação de Programas e Projetos de Saúde; Saúde Materno-Infantil; Sistema Único de Saúde

\section{Colaboradores}

R. M. S. M. Domingues e M. C. Leal participaram de todas as etapas da produção do artigo e foram responsáveis pela versão final. Z. M. A. Hartz participou da concepção, análise dos dados e aprovação da versão final. M. A. B. Dias colaborou na análise dos dados, na revisão crítica do conteúdo e aprovação da versão final.

\section{Referência}

1. Victora CG, Aquino EML, Leal MC, Monteiro CA, Barros FC, Szwarcwald CL. Maternal and child health in Brazil: progress and challenges. Lancet 2011; 377:1863-76

2. Carroli G, Rooney C, Villar J. How effective is antenatal care in preventing maternal mortality and serious morbidity? An overview of the evidence. Paediatr Perinat Epidemiol 2001; 15 Suppl 1:S1-42.

3. Villar J, Carroli G, Khan-Neelofur D, Piaggio G, Gülmezoglu M. Patterns of routine antenatal care for low-risk pregnancy. Cochrane Database Syst Rev 2001; (4):CD000934.

4. Barros FC, Bhutta ZA, Batra M, Hansen TN, Victora CG, Rubens CE; and the GAPPS Review Group. Global report on preterm and stillbirth (3 of 7): evidence for effectiveness of interventions. BMC Pregnancy Childbirth 2010; 10 Suppl 1:S3.

5. Fonseca SC, Coutinho ESF. Pesquisa sobre mortalidade perinatal no Brasil: revisão da metodologia e dos resultados. Cad Saúde Pública 2004; 20 Suppl 1:S7-19.
6. Silveira SD, Santos IS. Adequação do pré-natal e peso ao nascer: uma revisão sistemática. Cad Saúde Pública 2004; 20:1160-8.

7. Leal MC, Gama SGN, Ratto KMN, Cunha CB. Uso do índice de Kotelchuck modificado na avaliação da assistência pré-natal e sua relação com as características maternas e o peso do recém-nascido no Município do Rio de Janeiro. Cad Saúde Pública 2004; 20 Suppl 1:63-72.

8. Schoeps D, Almeida MF, Alencar GP, França Jr. I, Novaes HMD, Siqueira AAF, et al. Fatores de risco para mortalidade neonatal precoce. Rev Saúde Pública 2007; 41:1013-22.

9. Wehby GL, Murray JC, Castilla EE, Lopez-Camelo JS, Ohsfeldt RL. Prenatal care effectivenesse and utilization in Brazil. Health Policy Plan 2009; 24:175-88.

10. Debiec KE, Paul KJ, Mitchell CM, Hitti JE. Inadequate prenatal care and risk of preterm delivery among adolescents: a retrospective study over 10 years. Am J Obstet Gynecol 2010; 203:122.e1-6. 
11. Malta DC, Duarte EC, Escalante JJ, Almeida MF, Sardinha LM, Macário EM, et al. Mortes evitáveis em menores de um ano, Brasil, 1997 a 2006: contribuições para a avaliação de desempenho do Sistema Único de Saúde. Cad Saúde Pública 2010; 26:481-91.

12. Malta DC, Duarte EC, Almeida MF, Dias MAS, Morais Neto OL, Moura L, et al. Lista de causas de mortes evitáveis por intervenções do Sistema Único de Saúde do Brasil. Epidemiol Serv Saúde 2007; 16:233-4.

13. Kotelchuck M. An evaluation of the kessner adequacy of prenatal care index and a proposed adequacy of prenatal care utilization index. Am J Public Health 1994; 84:1414-20.

14. American College of Obstetricians and Gynecologists. Standards for obstetric-gynecologic services. Washington DC: American College of Obstetricians and Gynecologists; 1985.

15. Ribeiro ERO, Gimarães AMDN, Bettiol H, Lima DDF, Almedida ML, Souza L, et al. Risk factors for inadequate prenatal care use in the metropolitan area of Aracaju, Northeast Brazil. BMC Pregnancy Childbirth 2009; 9:31.

16. Silveira DS, Santos IS, Dias da Costa JS. Atenção pré-natal na rede básica: uma avaliação da estrutura e do processo. Cad Saúde Pública 2001; 17:131-9.

17. Carvalho DS, Novaes HMD. Avaliação da implantação de programa de atenção pré-natal no município de Curitiba, Paraná, Brasil: estudo em coorte de primigestas. Cad Saúde Pública 2004; 20 Suppl 2:S220-30.

18. Koffman MD, Bonadio IS. Avaliação da atenção pré-natal em uma instituição filantrópica da cidade de São Paulo. Rev Bras Saúde Matern Infant 2005; 5 Suppl 1:S23-32.

19. Ministério da Saúde. Programa de Humanização do Pré-Natal e Nascimento. Brasília: Secretaria de Políticas de Saúde; 2000.

20. Trevisan MR, De Lorenzi DRS, Araújo NM, Ésber K. Perfil da assistência pré-natal entre usuárias do Sistema Único de Saúde em Caxias do Sul. Rev Bras Ginecol Obstet 2002; 24:293-9.

21. Coutinho T, Teixeira MTB, Dain S, Sayd JD, Coutinho LM. Adequação do processo de assistência pré-natal entre as usuárias do Sistema Único de Saúde em Juiz de Fora, MG. Rev Bras Gineco Obstet $2003 ; 25: 717-24$

22. Serruya SJ, Cecatti JG, Lago TG. O Programa de Humanização no Pré-natal e Nascimento do Ministério da Saúde no Brasil: resultados iniciais. Cad Saúde Pública 2004; 20:1281-9.

23. Coutinho T, Monteiro MFG, Sayd JD, Teixeira MTB, Coutinho CM, Coutinho LM. Monitoring the prenatal care process among users of the Unified Health Care System in a city of the Brazilian Southeast. Rev Bras Ginecol Obstet 2010; 32:563-9.

24. Andreucci CB, Cecatti, JG. Desempenho de indicadores de processo do Programa de Humanização do Pré-Natal e Nascimento no Brasil: uma revisão sistemática. Cad Saúde Pública 2011; 27:1053-64
25. Ministério da Saúde. Pré-Natal e Puerpério: atenção qualificada e humanizada. Brasília: Ministério da Saúde; 2006.

26. Szwarcwald CL, Damacena GN. Amostras complexas em inquéritos populacionais: planejamento e implicações na análise estatística dos dados. Rev Bras Epidemiol 2008; 11 Suppl 1:38-45.

27. McLaughlin JA, Jordan GB. Logic models: a tool for telling your program's performance story. Eval Program Plann 1999; 22:65-72.

28. National Institute for Health and Clinical Excellence. Antenatal care: routine care for the healthy pregnant woman. Clinical guideline. http://www. nice.org.uk/nicemedia/live/11947/40145/40145. pdf (acessado em 12/Ago/2010).

29. Chrestani MAD, Santos IS, Cesar JA, Winckler LS, Gonçalves TS, Neumann NA. Assistência à gestação e ao parto: resultado de dois estudos transversais em áreas pobres das regiões Norte e Nordeste do Brasil. Cad Saúde Pública 2008; 24:1609-18.

30. Dias-da-Costa JS, Madeira ACC, Luz RM, Britto MAP. Auditoria médica: programa de pré-natal em posto de saúde na região Sul do Brasil. Rev Saúde Pública 2000; 34:329-36.

31. Silveira MF, Barros AJD, Santos IS, Matijasevich A, Victora CG. Socioeconomic differentials in performing urinalysis during prenatal care. Rev Saúde Pública 2008; 42:389-95.

32. Vettore MV, Dias M, Domingues RM, Vettore MV, Leal MD. Cuidados pré-natais e avaliação do manejo da hipertensão arterial em gestantes do SUS no Município do Rio de Janeiro, Brasil. Cad Saúde Pública 2011; 27:1021-34.

33. Domingues RMSM. A presença de um(a) acompanhante durante a atenção ao parto: a experiência da Maternidade Leila Diniz. In: Barbosa RM, Aquino EML, Heilborn ML, Berquó E, organizadores. Interfaces - gênero, sexualidade e saúde reprodutiva. Campinas: Editora da Unicamp; 2002. p. 279-307.

34. Leal MC, Gama SGN, Campos MR, Cavalini LT, Garbayo LS, Brasil CLP, et al. Fatores associados à morbi-mortalidade perinatal em uma amostra de maternidades públicas e privadas do Município do Rio de Janeiro, 1999-2001. Cad Saúde Pública 2004; 20 Suppl 1:S20-33.

35. Hodnett ED, Gates S, Hofmeyr GJ, Sakala C. Continuous support for women during childbirth. Cochrane Database Syst Rev 2007; (3):CD003766.

36. Caldeira AP, Fagundes GC, Aguiar GN. Intervenção educacional em equipes do Programa de Saúde da Família para promoção da amamentação. Rev Saúde Pública 2008; 42:1027-33.

37. Almeida SDM, Barros MBA. Atenção à saúde e mortalidade neonatal: estudo caso-controle realizado em Campinas, SP. Rev Bras Epidemiol 2004; 7:22-35.

38. Barros FC, Victora CG, Barros AJ, Santos IS. The challenge of reducing neonatal mortality in middle-income countries: findings from three Brazilian birth cohorts in 1982, 1993, and 2004. Lancet 2005; 365:847-54.

Recebido em 18/Jul/2011

Versão final reapresentada em 11/Set/2011

Aprovado em 20/Out/2011 